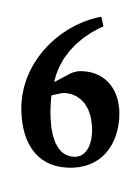

\title{
LA PERCEPCIÓN DEL DOCENTE UNIVERSITARIO ANTE EL USO Y VALOR DE LOS DATOS ABIERTOS
}

\author{
(THE PERCEPTION OF THE UNIVERSITY PROFESSOR REGARDING \\ THE USE AND VALUE OF OPEN DATA)
}

Begoña Rivas-Rebaque

Universidad Rey Juan Carlos

Felipe Gértrudix-Barrio

Julio César de Cisneros de Britto

Universidad de Castilla-La Mancha

DOI: 10.5944/educXX1.21317

Cómo referenciar este artículo/How to reference this article:

Rivas-Rebaque, B.; Gértrudix-Barrio, F. y de Cisneros de Britto, J.C. (2019). La percepción del docente universitario ante el uso y valor de los datos abiertos. Educación XX1, 22(2), 141-163, doi: 10.5944/educXX1.21317

Rivas-Rebaque, B.; Gértrudix-Barrio, F. \& de Cisneros de Britto, J.C. (2019). The perception of the University Professor regarding the use and value of Open Data. Educación XX1, 22(2), 141-163, doi: 10.5944/educXX1.21317

\section{RESUMEN}

El avance de la conciencia democrática junto al aumento de población está provocando la necesidad de ofrecer a la sociedad datos que los propios gobiernos se obligan legalmente, y que los ciudadanos necesitan cada vez más. Muchos de estos datos son ofrecidos en modo abierto; son los denominados datos abiertos (Open Data por sus siglas en inglés, OD), y que son actualidad, futuro, apertura, transparencia, conocimiento, eficiencia, ahorro de tiempo, profundidad en la investigación, desarrollo en la educación, precisión en los análisis y por tanto en las patologías y diagnósticos de cualquier ámbito de nuestra vida que interese estudiar. No obstante, de poco o nada sirve conocer todo sobre los mismos si no sabemos qué hacer con ellos ni tenemos la capacidad para explotarlos. En este contexto, y ante las escasas iniciativas 
en el entorno educativo, se presenta el siguiente trabajo que tiene como objeto de estudio los datos abiertos dentro del ámbito de las Facultades de Educación españolas, intentando dar respuesta a si se pueden aprovechar en la enseñanza superior como recurso docente. Como objetivo principal se plantea conocer qué tipo de percepción poseen los docentes universitarios ante el uso y el valor de los datos abiertos para el proceso de enseñanzaaprendizaje. La muestra ha sido de 192 docentes, con un margen de error máximo aplicable a datos globales no segmentados con un nivel de confianza del $95,5 \%$, que en el caso más desfavorable $(\mathrm{p}=\mathrm{q}=50 \%)$, es de $\pm 7,2 \%$. Para ello, se ha elaborado un diseño de investigación no experimental, de tipo exploratorio, utilizando el instrumento del cuestionario para la recogida de información. Los resultados demuestran que el uso de datos abiertos en Educación Superior obliga a una mejora de las políticas educativas que impulse la alfabetización digital, fomentando con ello el empoderamiento educativo.

\section{PALABRAS CLAVE}

Datos abiertos; empoderamiento; alfabetización digital; docentes; educación superior.

\section{ABSTRACT}

The advance of democratic awareness along with the increase in population is provoking the need to offer the society data that our own governments are legally obliged to provide and that citizens need more and more. Much of this data is offered in open mode; the so-called open data (OD), which is current, future, openness, transparency, knowledge, efficiency, time saving, depth of research, development in education, precision analysis and therefore in the pathologies and diagnoses of any area of our life that is of interest to study. However, it is of little or no use to know all about it if we do not know what to do with it or have the capacity to exploit it. In this context, and faced with the scarce initiatives in the educational field, this study is presented with the objective of studying open data within the scope of the Spanish Faculties of Education, aiming to learn whether they can be used in higher education as a teaching resource. The main objective is to know what type of perception university professors have regarding the use and value of open data for the teaching-learning process. The sample was of 192 teachers, with a maximum margin of error applicable to non-segmented global data with a confidence level of $95.5 \%$, which in the worst case $(\mathrm{p}=\mathrm{q}=50 \%)$ is $\pm 7.2 \%$. A non-experimental research design has been developed, of an exploratory type, using the instrument of the 
questionnaire for the collection of information. The results demonstrated that use in higher education requires an improvement of educational policy that promotes digital literacy thus promoting educational empowerment.

\section{KEYWORDS}

Open data; empowerment; digital literacy; teachers; higher education.

\section{INTRODUCCIÓN}

\section{La vorágine de los datos}

Conocedores del contexto actual de la sociedad del conocimiento donde se mueven cantidades ingentes de datos a diario, no podría entenderse la adquisición de los conocimientos y la comunicación sin una necesaria, continua y vertiginosa relación a través de la interacción con los datos. En consecuencia, el uso de términos como "Open Data» o «Big Data» (datos masivos) se están haciendo familiares debido a su gran volumen, a la rapidez en su producción, a la multiplicidad de formatos, a la calidad y procedencia de los mismos (IBM Analytics, s/f), a su validez, a la variabilidad dependiendo del contexto, a su gran volatilidad, a su seguridad; a su comprensión y legibilidad, así como al valor que aportan a la sociedad (Normandeau, 2013, Rijmenam, 2013 y Firican, 2017).

Por todo ello, la apertura, el acceso, el análisis y el tratamiento de los datos son aspectos a tener muy en cuenta en la actualidad y en especial en el ámbito de la educación superior.

\section{Antecedentes de la apertura de datos en el contexto educativo}

Desde el año 2012, la Universidad Abierta de Reino Unido empezó a conceder gran relevancia al acceso abierto de datos (Open Access, OA) desarrollando un modelo de educación a distancia flexible. Este término dio lugar a diversas expresiones, todas ellas relacionadas con educación: Open source, Open Educational Resources (OER), Open Courses, Open Research, Open Data (OD), Open APIs (Application Programme Interface), Interfaz de Programación de Aplicaciones (IPA), Open Access Publishing (Weller, 2012).

Dentro del contexto educativo se debe hacer referencia a los recursos educativos en abierto (REA), ya que al igual que los datos abiertos provienen 
del contexto de la cultura del código abierto, aunque es conveniente especificar que se trata de contenidos en abierto. De manera más detallada, la UNESCO (2008) fue la primera organización que recogió este término y los define como: «materiales pedagógicos, de investigación y de aprendizaje que se encuentran en el dominio público y tienen licencia abierta, como Creative Commons» (pág. 2). Adicionalmente, y como explican Neil Butcher, Asha Kanwar y Stamenka Uvalic-Trumbic (2015), dado que en Educación Superior se utilizan publicaciones científicas como materiales para dichos estudios, se debe hacer la distinción entre este tipo de publicaciones de acceso libre y los REA, que surgen como una revolución de la enseñanza y el aprendizaje y contribuyen a hacer la educación más accesible, sobre todo, en aquellas partes donde los recursos para el aprendizaje escasean por problemas económicos (Open Society Foundations, 2007).

En cuanto a los procesos de aprendizaje mediante el uso de contenidos abiertos, y siguiendo los objetivos planteados en Horizon 2010, no solo se transmite material educativo de manera gratuita, sino que también se adquieren competencias, se requieren habilidades de búsqueda, evaluación y reutilización de los mismos (Browne, Holding, Howell \& Rodway-Dyer, 2010). Desde 2015, se aprecia un importante crecimiento de los REA en la educación superior a nivel internacional, situando a los materiales de cursos abiertos (Open CourseWare-OCW) como los promotores de estos recursos a nivel mundial en el «desarrollo e intercambio de materiales libremente disponibles, autónomos para cursos y enseñanza online» (Butcher, Kanwar \& Uvalic-Trumbic, 2015, pág. 73).

En este sentido, Cabero y Llorente (2008) toman como ejemplo de experiencia donde pueden interactuar profesores y estudiantes los Open Course Ware argumentando que «cada vez tendremos acceso libre a información de más calidad» (pág. 3). Del mismo modo, cabe señalar iniciativas como las de Vancouver (British Columbia) sobre los libros de textos abiertos (Open textbooks) ya que podrían ser modificados por los docentes adaptándose al contexto (Klassen, 2013) o el movimiento OER como ejemplos evidentes de proyectos de aprendizaje abierto donde «el acceso global al conocimiento y la democratización de la educación son factibles» (Mireles, 2012, pág. 82).

\section{Definición, principios y utilidad de los datos abiertos en educación}

El origen de la filosofía de los datos abiertos tuvo lugar en 2007, a raíz de la afirmación de Tim O’Reilly en la conferencia OSCON: «los datos abiertos en realidad podrían ser más importantes que el código abierto» (Muñoz Santamaría, 2016). Posteriormente, en el 2009, el gobierno de los 
Estados Unidos (proyecto data.gov) lanzó su catálogo de datos, idea que recogió el Reino Unido en 2010 dando a conocer su portal de Open Data y así se fue extendiendo esta iniciativa al resto de países. Así, Open Knowledge Foundation señala que «los datos abiertos pueden ser utilizados, reutilizados y redistribuidos libremente por cualquier persona, y que se encuentran sujetos, cuando más, al requerimiento de atribución y de compartirse de la misma manera en que aparecen» (Open Knowledge International, s/f).

En lo que respecta al marco legislativo sobre los datos abiertos, a nivel europeo, se rige por la Directiva 2003/98/CE del Parlamento Europeo y del Consejo sobre el acceso y la reutilización de la información del sector público, y para el ámbito estatal la reutilización de los datos públicos la legislación actual se basa en la Ley 37/2007.

Como ya hemos indicado en trabajos anteriores los « 8 principios del Open Data» que, según Tauberer (s/f), deben contemplar dichos datos para que se consideren como tal y el formato en el que deben ser publicados para que puedan ser reutilizados por terceros son: 1. Públicos; 2. Datos en bruto; 3. Actualizados; 4. Accesibles; 5. Estructurados; 6. Sin registro; 7. Abiertos; 8. Libres (Rivas Rebaque, 2018).

En el ámbito educativo, los datos abiertos salen de las instituciones educativas pudiendo ser explotados y/o utilizados por ellas, además de ser datos de investigación. Además, estos datos pueden ser clasificados como: a) datos abiertos de la educación, b) datos o recursos educativos abiertos (REA) y c) datos abiertos para la educación (Guy, 2014). El tipo de datos que se pueden encontrar en las instituciones académicas son: datos del estudiante, datos curriculares, datos institucionales, datos generados por los usuarios como datos de rendimiento y métricas de aprendizaje o datos gubernamentales (Wikibooks, 2014).

Profundizando en el uso de los datos abiertos en la Educación Superior, el informe elaborado por McKinsey Global Institute (MGI) (Manyika, Chui, Farrell, Van Kuiken, Groves \& Almasi Doshi, 2013) identifica cinco herramientas (palancas) que pueden favorecer el valor potencial de los datos abiertos en educación y que son: 1) Mejoraría la instrucción al obtener información sobre los estilos de aprendizaje y el rendimiento de los estudiantes; 2) Ayudaría a una mejor adaptación de los estudiantes a los planes de estudio al poder seleccionar las opciones educativas que coincidan con los intereses del alumnado; 3) Permitiría una adecuación de los estudiantes al empleo al relacionar las habilidades requeridas por las empresas con las destrezas que poseen los estudiantes; 4) Transparencia en la educación que podría ayudar en la toma de decisiones a la hora de elegir centros educativos; y 5) Administración más eficiente, ya que conseguiría 
una reducción significativa de los precios pagados por los suministros y servicios.

\section{Estado de la cuestión}

En el campo de las experiencias educativas encontramos iniciativas exitosas como las de OpenCoesionSchool (2013), proyecto orientado a la Educación Secundaria y desarrollado en Italia, en la que se promueve la ciudadanía activa y el desarrollo de la conciencia cívica; el Curso ProyectarExplorar-Narrar (Núcleo Interdisciplinario sobre Recursos Educativos Abiertos y Accesibles, 2016), iniciativa de la Universidad de la República de Uruguay, dirigido a universitarios para crear REAs utilizando OD como herramienta para la construcción de conocimiento crítico; el espacio web QEdu (s/f) de Brasil que contiene los principales datos de la enseñanza básica de ese país con objeto de ayudar a gestores, directores, profesores y todos los interesados a hacer mejores elecciones en la educación; Mejora tu Escuela de México (2015), es una plataforma de participación ciudadana para transformar la educación en México. Además, existen colectivos cuya actividad está vinculada a datos abiertos entre las que se encuentran: Catalunya Dades (2014); Civio (2015); Novagob (2017) y OpenKratio (2018) y en las que se ofrece la posibilidad de conocer cómo reutilizar los datos que las administraciones ponen a nuestro servicio (Datos.gob.es, 2014).

Por otro lado, cada vez existe una mayor literatura en torno al uso de datos abiertos en distintos campos de investigación: sobre políticas de datos abiertos (Zuiderwijk y Janssen, 2014); toma de decisiones y rendición de cuentas (Baack, 2015); iniciativas gubernamentales a la hora de impulsar el uso de datos abiertos entre los ciudadanos (Hellberg y Hedström, 2015); sobre los procesos de transparencia (Umbrich, Neumaier y Polleres, 2015, Worthy, 2015); la apertura de los datos de investigaciones (Rouder, 2016); sobre el impacto en países en desarrollo (Davies y Perini, 2016); en la salud como datos médicos compartidos (Kostkova et al., 2016); o en la actividad turística (Pantano, Priporas y Stylos, 2017). Igualmente, en el ámbito científico, el recurso del vídeo como fuente de datos abiertos para la investigación (Gilmore, Adolph, Millman, \& Gordon, 2016).

En lo referente al contexto educativo, descubrimos diferentes trabajos que han abordado temáticas como el Linked Open Data y el desarrollo de aplicaciones (McAuley, Rahemtulla, Goulding \& Souch, 2011), el Linked Open Data utilizando los REAs (Mikroyannidis, Domingue, Maleshkova, Norton \& Simperl, 2016; Chicaiza Espinosa, 2016; Daga, D’Aquin, Adamou, \& Brown, 2016; Hyvönen et al., 2016); el Open data y Big data como beneficio y calidad de servicios de las administraciones (Mejía, 2014); el Open Data 
para el proceso de alfabetización mediática y competencia digital (Gálvez de la Cuesta, 2015); el uso de datos abiertos para el desarrollo de competencias profesionales (Gertrudis-Casado, Gértrudix-Barrio y Álvarez-García, 2016).

\section{MATERIAL Y MÉTODOS}

\section{Objetivos}

La finalidad de este trabajo es conocer la percepción del docente universitario ante el uso y el valor que ofrecen los datos abiertos como recursos didácticos dentro del ámbito de las Facultades de Educación de España. Para ello, se intenta dar respuesta a la pregunta de si el uso de los datos abiertos supone un aprovechamiento significativo para una mejora en la adquisición del conocimiento en el proceso de enseñanza y aprendizaje. A partir de este objetivo principal se derivan los siguientes objetivos secundarios:

- Investigar, bajo la perspectiva del docente, si existe alguna limitación en los planes de estudio de la formación del profesorado para el uso de estos recursos.

- Conocer la mejor forma de utilizar OD para generar información de valor añadido desde la perspectiva del docente.

- Indagar las ventajas y limitaciones del uso didáctico de los datos abiertos.

\section{Diseño de investigación}

La investigación se encuentra dentro del paradigma del diseño de investigación no experimental, de tipo exploratorio, observando el fenómeno tal y como se presenta sin manipulación de variables ni comparación de grupos de sujetos (Baena Paz, 2014; Abero, Berardi, Capocasale, García Montejo y Rojas Soriano, 2015). El cuestionario ha sido el instrumento para la recogida de información, el cual ha permitido integrar y estandarizar el proceso de recopilación de datos sobre las variables que se han medido y que han hecho posible conocer la situación actual sobre el conocimiento y la utilidad de los datos abiertos en las Facultades de Educación de España, bajo la mirada del docente universitario (Aguilar y Barroso, 2015).

La investigación se ha desarrollado durante el período de 2016-2017, ejecutándose en cinco fases de trabajo: a) análisis de la situación o problema, b) desarrollo de soluciones a partir de revisión de la literatura sobre datos abiertos en educación, c) definición de las unidades de análisis: diseño del instrumento, d) especificar el sistema de codificación: validación del 
instrumento y e) extraer las inferencias resultantes del análisis (De Benito Crosetti y Salinas Ibáñez, 2016).

\section{Muestra}

La unidad de análisis de la población de referencia está representada por docentes de universidad con la característica específica de ser profesores en las Facultades de Educación españolas, con un total de 1.409 docentes. En este sentido, el tamaño la muestra está directamente relacionada con la precisión de estimación que se ha realizado y con el grado de seguridad que se ha obtenido (Cosculluela, Fornieles y Turbany, 2009).

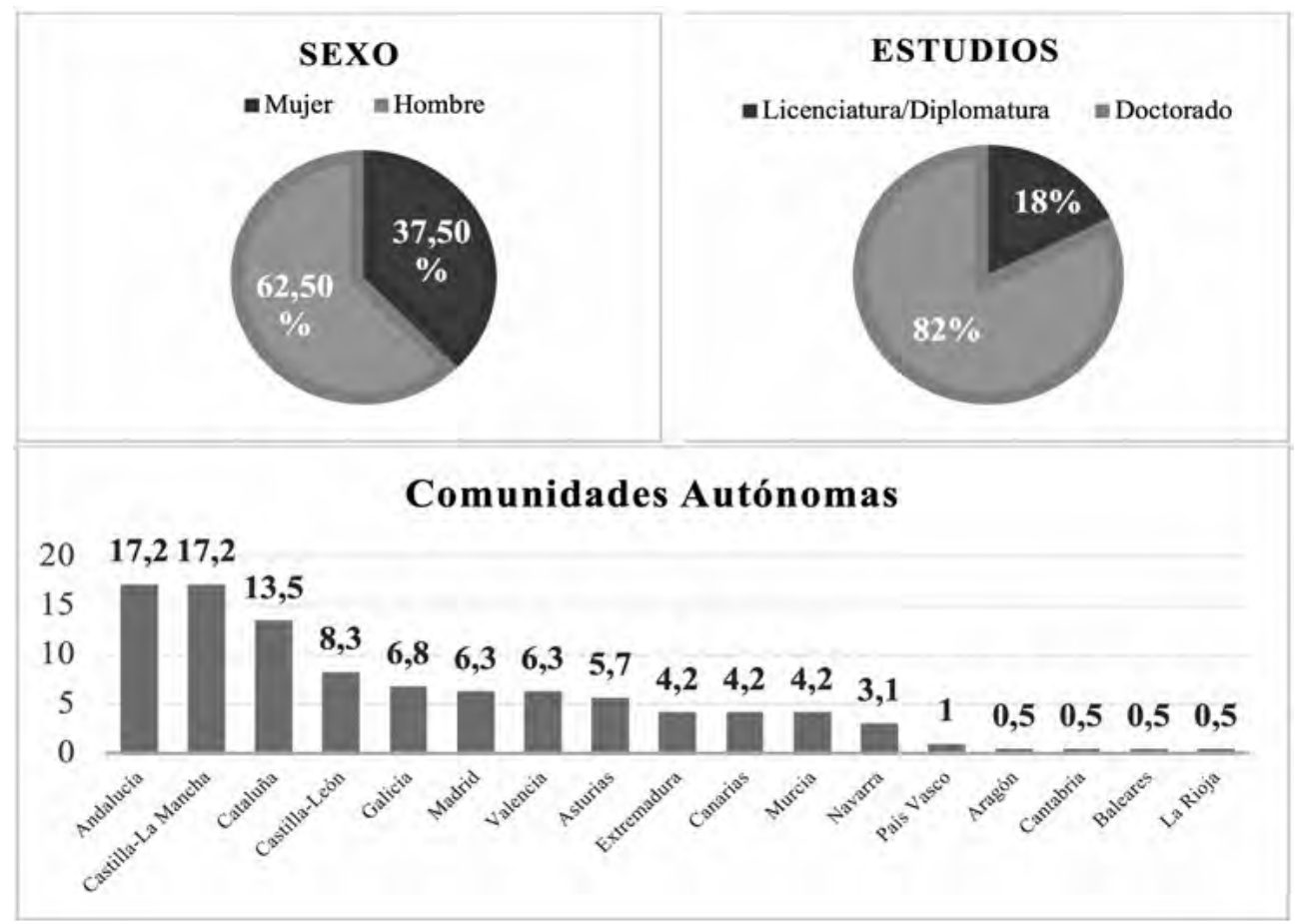

Gráfico 1. Descripción de la muestra

Fuente: elaboración propia

En lo que respecta a la selección de la muestra, se trata de un muestreo no probabilístico, dadas las características de la investigación (investigación educativa), y la elección de manera intencional de las unidades de la muestra (docentes de las Facultades de Educación). Además, el tipo de muestreo que 
mejor se ajusta a la investigación es el de bola de nieve, pues se localizan a algunos individuos, los cuales conducen a otros, y estos a otros, y así hasta conseguir una muestra suficiente (Bisquerra, 2004, págs. 145-149). Así, el tamaño de la muestra es de 192 docentes, 62,5\% de mujeres y 37,5\% de hombres, con un margen de error máximo aplicable a datos globales no segmentados con un nivel de confianza del $95,5 \%$ en el caso más desfavorable $(\mathrm{p}=\mathrm{q}=50 \%)$, es de $\pm 7,2 \%$. En el gráfico 1 se puede observar la descripción de la muestra obtenida atendiendo al sexo, nivel de estudios y perfil docente universitario.

\section{Instrumento}

El cuestionario ha sido el instrumento utilizado en la investigación. Su construcción se ha realizado a partir del análisis de los datos abiertos y el cruce con tres variables: la competencia digital (Ananiadou \& Claro, 2009; Area y Pessoa, 2012; Reig y Vílchez, 2013; Bellanca \& Brandt 2010), el empoderamiento ciudadano (Tejedor y Pulido, 2012; Álvarez-García, Gértrudix-Barrio y Rajas-Fernández, 2014) y las políticas educativas (Insight, 2011; Rivas-Rebaque, De Cisneros de Britto, Gértrudix-Barrio, 2015).

Para su validación se ha utilizado el método Delphi con tres oleadas en las que participaron nueve expertos, los cuales han analizado la univocidad, pertinencia y relevancia de las preguntas del cuestionario y ratificado la definición del constructo teórico del contenido de cada uno de los ítems. Para el análisis de las respuestas del Delphi, siguiendo a Astigarraga (2003), se ha optado por el estadístico de la media central. Mediante pruebas psicométricas, se ha sometido el cuestionario a pruebas de fiabilidad de consistencia interna, dando como resultado un 0.924 en la prueba de Alfa de Cronbach.

El cuestionario definitivo (https://goo.gl/fwKQer) ha quedado estructurado por 12 preguntas multirespuesta con preguntas cerradas precodificadas usando escala tipo Likert de 5 niveles y en abanico, 3 preguntas no estructuradas, un apartado con datos del perfil del encuestado sobre género, nivel de estudios, ocupación y la comunidad autónoma donde imparte la docencia, un vídeo explicativo sobre datos abiertos y la definición de los mismos a modo de introducción sobre la temática de los datos abiertos. Ha sido administrado a través de la herramienta de creación de formularios Google Forms y se ha utilizado como medio de difusión el correo electrónico. 


\section{Análisis de los datos}

Una vez validado el cuestionario final, se ha procedido a elaborar un directorio de correos electrónicos con la identidad de docentes de las distintas Facultades de Educación públicas de España y de las siguientes titulaciones: Grado en Magisterio en Educación Infantil y Educación Primaria; Grado en Educación Social y Grado en Pedagogía.

Todas las encuestas que se han realizado fueron anónimas y codificadas, empleando el paquete estadístico SPSS ( «Statistical Package for the Social Sciences», versión 23). En las preguntas de escala, en lugar de extraer la media y la desviación típica se ha optado por calcular el porcentaje de las respuestas más positivas y negativas (T2B y B2B), ya que proporcionaban una información más estricta, teniendo en cuenta que la concentración de respuestas en torno a determinadas posiciones de la escala hacía la media poco representativa. Teniendo en cuenta las variables sociodemográficas se ha establecido una comparación de medias (prueba t de Student) con el fin de comprobar la existencia de diferencias significativas entre dichas variables y las respuestas de las otras preguntas.

\section{RESULTADOS}

\section{Conocimiento y uso de los Open Data}

En primer lugar nos fijamos en el dato representativo sobre el alto porcentaje de profesorado $(63,5 \%)$ que declara un conocimiento en el uso de datos abiertos, si bien más de la mitad es por un motivo laboral, en detrimento de su potencial formativo con una menor significatividad.

En cuanto al acceso y uso de datos en abierto, no se observan diferencias significativas en función del sexo, el nivel de estudios o la ocupación. Sí se aprecia una pequeña diferencia en cuanto a los motivos de uso: los hombres utilizan en mayor medida los datos en abierto (51\% vs $36,6 \%$ ), para cuestiones no relacionadas con su trabajo.

En lo referente a las características específicas de los datos abiertos, los docentes se muestran muy favorables al papel de los datos abiertos como instrumento de empoderamiento educativo $(80 \%)$, que deben incorporarse en la formación universitaria de los estudiantes (82\%) e incorporarse en los planes de estudio (74\%). En consecuencia, ambos aspectos permitirían utilizar los datos abiertos como complemento de formación en la actividad académica, bien como instrumento para la docencia o como recurso educativo (ver gráfico 2). 


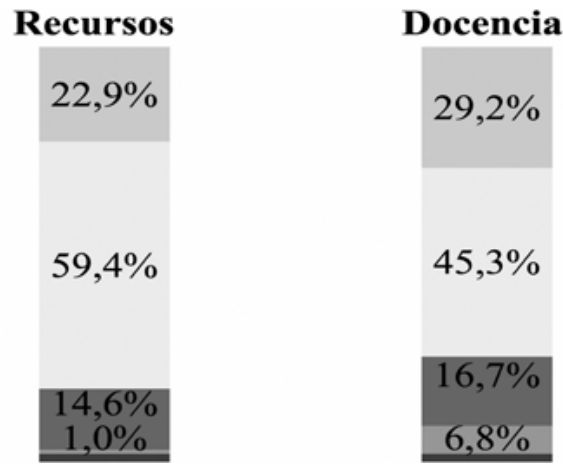

Gráfico 2. Características específicas de los datos abiertos

Fuente: elaboración propia

\section{El uso de los datos abiertos en la educación universitaria}

Una amplia mayoría de docentes (89\%) ve la necesidad de una formación tanto específica como transversal a la hora de utilizar los datos abiertos. De manera específica se debe enseñar a comprobar la calidad de los datos, tener un buen juicio en la selección, calidad, validez y fiabilidad de la información antes que a la investigación, de tal forma que sean orientados al fomento del pensamiento crítico. Asimismo, a la cuestión sobre cuál es la mejor forma, para el estudiante, de utilizar los datos en abierto para generar información de valor añadido, casi un $70 \%$ del profesorado considera que es tan conveniente la creación de un juicio crítico personal del estudiante (análisis personal > construcción del conocimiento) como la producción de la información, a partir de un criterio de un especialista (análisis provenientes $>$ conocimiento ya creado).

En lo que respecta a las ventajas del uso de datos abiertos con respecto a otros materiales, se observa que el profesorado está de acuerdo en las ventajas del uso de datos abiertos frente a los libros de texto en un $70 \%$ y con respecto a la comparativa con la prensa en un $60 \%$. Sin embargo, este porcentaje disminuye de manera considerable frente a las opiniones de expertos o a los datos provenientes de los artículos en revistas científicas, con casi un $40 \%$ y un $34 \%$, respectivamente (ver Tabla 1 ). 
Tabla 1

Ventajas de los datos abiertos

\begin{tabular}{|c|c|c|c|c|c|c|c|c|c|}
\hline \multirow{2}{*}{$\begin{array}{c}\% \mathbf{T} 2 B \\
\text { (Totalmente de } \\
\text { acuerdo + de } \\
\text { acuerdo) }\end{array}$} & \multicolumn{2}{|c|}{ Sexo } & \multicolumn{2}{|c|}{ Estudios } & \multicolumn{3}{|c|}{ Ocupación } & \multicolumn{2}{|c|}{$\begin{array}{c}\text { Conocimiento } \\
\text { y uso } \\
\text { open data }\end{array}$} \\
\hline & 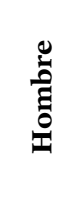 & 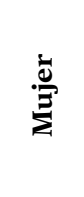 & $\begin{array}{l}\frac{0}{\pi} \\
\frac{\pi}{0} \\
\frac{0}{0} \\
0\end{array}$ & 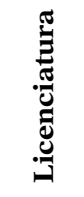 & 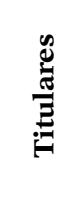 & 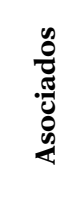 & 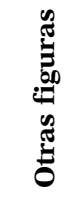 & 的苞 & 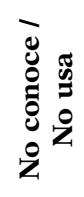 \\
\hline Base: & 72 & 120 & 34 & 158 & 80 & 76 & 36 & 122 & 70 \\
\hline Libro de texto & 68,1 & 71,7 & 64,7 & 71,5 & 70,0 & 72,4 & 66,7 & 65,6 & 78,6 \\
\hline Prensa & 61,1 & 60,0 & 50,0 & 62,7 & 62,5 & 64,5 & 47,2 & 56,6 & 67,1 \\
\hline Expertos & 37,5 & 40,8 & 32,4 & 41,1 & 46,3 & 40,8 & 22,2 & 35,2 & 47,1 \\
\hline Revistas & 33,3 & 34,2 & 38,2 & 32,9 & 36,3 & 39,5 & 16,7 & 29,5 & 41,4 \\
\hline
\end{tabular}

Nota: diferencias significativas al 95\% (t-student)

Tabla 2

Aspectos negativos de los datos abiertos

\begin{tabular}{|c|c|c|c|c|c|c|c|c|c|}
\hline \multirow[b]{2}{*}{$\begin{array}{c}\% \mathrm{~T} 2 \mathrm{~B} \\
\begin{array}{c}\text { (Totalmente de acuerdo }+ \\
\text { de acuerdo })\end{array}\end{array}$} & \multicolumn{2}{|c|}{ Sexo } & \multicolumn{2}{|c|}{ Estudios } & \multicolumn{3}{|c|}{ Ocupación } & \multicolumn{2}{|c|}{$\begin{array}{l}\text { Conocimiento } \\
\text { y uso open } \\
\text { data }\end{array}$} \\
\hline & : & $\stackrel{\grave{\leftrightarrows}}{\stackrel{\Xi}{\Xi}}$ & 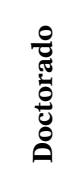 & 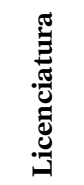 & 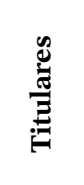 & $\frac{2}{\frac{\pi}{\pi}}$ & 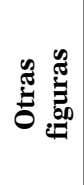 & 的 & 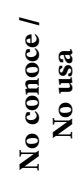 \\
\hline Base: & 72 & 120 & 34 & 158 & 80 & 76 & 36 & 122 & 70 \\
\hline $\begin{array}{l}\text { Acceso sin las competencias } \\
\text { requeridas }\end{array}$ & 62,5 & 63,3 & 64,7 & 62,7 & 66,3 & 61,8 & 58,3 & 67,2 & 55,7 \\
\hline $\begin{array}{l}\text { Uso sin una perspectiva crítica } \\
\text { y un análisis del contexto }\end{array}$ & 84,7 & 84,2 & 73,5 & 86,7 & 88,8 & 77,6 & 88,9 & 87,7 & 78,6 \\
\hline $\begin{array}{l}\text { Uso inadecuado y seleccionar- } \\
\text { los de manera incorrecta }\end{array}$ & 76,4 & 80,8 & 79,4 & 79,1 & 77,5 & 80,3 & 80,6 & 82,0 & 74,3 \\
\hline $\begin{array}{l}\text { Uso como herramienta predic- } \\
\text { tiva }\end{array}$ & 43,1 & 55,8 & 58,8 & 49,4 & 48,8 & 50,0 & 58,3 & 55,7 & 42,9 \\
\hline Largo proceso de aprendizaje & 29,2 & 33,3 & 26,5 & 32,9 & 32,5 & 32,9 & 27,8 & 26,2 & 41,4 \\
\hline
\end{tabular}

Nota: diferencias significativas al 95\% (t-student)

Por lo que se refiere a los aspectos negativos, un 63\% considera que un mal uso de datos en abierto, sin las competencias requeridas y sin una 
perspectiva crítica, es contraproducente en la educación superior, por lo que se debe contar con una disposición crítica a la hora de manejar los datos abiertos (84\%), ya que se puede tender a una selección inadecuada de ellos $(79 \%)$ y convertirse en un recurso prescriptivo. Los encuestados que no conocen o no han usado datos abiertos consideran que necesitarían bastante tiempo para aprender a usarlos (Ver Tabla 2).

\section{Usos didácticos de los datos abiertos}

En lo concerniente a las actividades y usos didácticos para aprovechar los datos abiertos, la mayoría de los encuestados coinciden en la relevancia de su uso para actividades docentes como: analizar temas actuales (94\%), elaborar supuestos prácticos y desarrollar contenidos (91\%), siendo las que menor apoyo obtienen aquellas actividades que tienen que ver con la creación o aplicación de tecnologías (53\%) (ver figura 3). Esto indica que las actividades que incluyen manipulación de herramientas técnicas están consideradas menos esenciales que aquellas que desarrollan el pensamiento crítico y analítico, y el aprendizaje autónomo.

\section{Tabla 3}

Actividades y usos didácticos de los datos abiertos

Usos didácticos de los datos abiertos

Analizar temas actuales en clase

$94 \%$

Elaborar supuestos prácticos basados en datos en abierto

$91 \%$

Desarrollar contenidos

$83 \%$

Integrar el uso de herramientas de visualización de datos

$78 \%$

Incluir formas de hacer datos interactivos

Crear aplicaciones para el aula

Añadir tecnologías como realidad virtual para buscar inmersividad en los datos

Nota: algunos docentes también mencionaron: fundamentar opiniones, fomento pensamiento crítico, búsqueda y selección información, aprendizaje continuo y colaboración/difusión

Sobre las cualidades con las que debería contar un profesor para el uso docente de los datos abiertos, se refleja una actitud positiva en cuanto a disposición y uso de datos abiertos dado el consenso de la mayoría de los encuestados. Otro dato, a tener en cuenta, es la elección de los profesores titulares que consideran relevante contar con una buena actitud, mientras 
que para los profesores asociados es conocer sitios de búsqueda y manejar herramientas de visualización de contenidos. Este aspecto quizá puede deberse al proceso de adaptación que cada uno de ellos ha debido asimilar para su propio aprendizaje.

$\mathrm{Al}$ respecto de las labores docentes para que su utilización produzca una comprensión lógica de la información entre el alumnado, se deduce que lo más relevante es fomentar la actitud crítica ante el uso de datos abiertos e incorporar a la labor docente el uso y manejo de este tipo de datos, considerando el aspecto técnico como un requisito destacado para ser un profesional cualificado.

\section{Mejora en el aprendizaje con el uso de datos abiertos}

Se concede mucha importancia al fomento de un pensamiento crítico a través del uso de datos en abierto, así como el manejo de los datos, de su selección, análisis y explotación. Mientras que para los profesores titulares, es significativamente importante el objetivo de fomentar una perspectiva crítica y selectiva ante la información facilitada por los medios, entre los docentes asociados se destaca el hecho de alcanzar mayores competencias, más profundidad de análisis y elaborar trabajos más serios.

En cuanto a la evaluación de tareas, se observa que, si bien todos los criterios de evaluación ofertados son considerados mayoritariamente relevantes o muy relevantes, existe una clara preferencia por aquel que insiste en la reflexión frente al manejo técnico de la información. Igualmente, se deduce un consenso respecto de aquellos criterios de evaluación que coinciden en reelaborar contenidos, evaluarlos y referenciarlos adecuadamente. Por tanto, siguen primando las funciones cognitivas a las habilidades técnicas, pero se apuntan conatos de cambios en el plano de la integración y reelaboración de contenidos.

\section{DISCUSIÓN Y CONCLUSIONES}

La falta de uso de los datos abiertos en la actividad académica y el desconocimiento de estos por parte de algunos de los docentes universitarios indica la necesidad de adquirir una mayor información sobre estos recursos para su posterior utilización: es conveniente instruir en la «cultura de reutilización» (Díez y Millán, 2011). La academia sigue apostando por contenidos cerrados difícilmente adaptables al mundo laboral al que se enfrentará el egresado universitario. Tiene que ir permutando desde una educación de "hardware» hacia una educación de «software» (Torres 
Menárguez, 2017b) y orientada a desarrollar el pensamiento crítico. Esta alternancia podrá lograrse con una flexibilización del currículo, una formación del profesorado adaptada a los nuevos entornos de enseñanza-aprendizaje, una reestructuración de los espacios que promueva nuevas metodologías y mejore el rendimiento académico, y un aumento en el presupuesto destinado por las universidades para el avance de los servicios tecnológicos (Iglesias, 2016). Y es en ese ecosistema, cuando el uso de datos abiertos habilita a pensar, investigar, resolver problemas, afrontar retos, contrastar opiniones, etc., en suma, a enseñanzas para la vida (Sánchez, 2016).

Como respuesta al interrogante sobre la viabilidad de esta transformación educativa, conviniendo con Torres Menárguez (2017a), se debe comenzar con grupos pequeños como los directores de centros y los docentes que estén a favor del cambio de aprendizaje; es decir, desde las escuelas como institución local. De manera similar, se pronuncia Giráldez (2017) apuntando que existen diferentes grupos de profesores, unos decididos a innovar y otros a continuar con la enseñanza tradicional y cuestiona si el docente, bajo su responsabilidad personal, está dispuesto a cambiar la escuela.

En cualquier caso, en el contexto universitario, no se debe olvidar que el docente cuenta con el derecho de la libertad de enseñar en el ejercicio de su libertad de cátedra en la que puede establecer las metodologías y estrategias de enseñanza que considere pertinentes. Sin embargo, se debe resaltar la oportunidad que supone para cualquier docente el apostar por esos cambios educativos, ya sea como un logro personal -motivación intrínseca一, o como medida de presión social —motivación extrínseca一, transformando su rol de docente hacia el de mentor, promoviendo la cultura de la exploración y la colaboración: desde la pedagogía a la heutagogía en el sentido de un aprendizaje que se ha de producir al mismo tiempo que se investiga (Hase \& Kenyon, 2007).

Del mismo modo, coincidimos con las afirmaciones de Magro en SantillanaLab (sf) al indicar que se debe trabajar por la transformación y no por la transmisión. En este sentido, la tarea del docente no debe centrarse en clases magistrales (Sage of the stage) sino que se debe guiar y orientar hacia el proceso de enseñanza-aprendizaje (Guide on the side) (Zapata Rojas, 2013), procurando un escenario viable para la implementación de datos abiertos como recursos educativos, ya que su función es insustituible a la hora de contrastar la veracidad de los datos y de saber manejar la información con criterio. El docente debe apoyar a los estudiantes para evitar prejuicios y falacias cognitivas, ya que tener acceso a un volumen amplio de datos, en ocasiones, va a entrañar mayor dificultad a la hora de seleccionar la información adecuada y va a requerir de habilidades y 
destrezas específicas para el análisis y explotación de los datos, incluso para el manejo de determinadas herramientas informáticas.

En definitiva, se hace necesario dar un nuevo impulso a la alfabetización digital con el fin de fomentar un empoderamiento educativo y participativo (Gértrudix-Barrio, Gálvez de la Cuesta, Said-Hung \& DuránMedina, 2016), puesto que para ser «ciudadanos digitales» se requiere de una adecuada formación donde se instruya a ciudadanos con criterio para desenvolverse en la aldea digital (Cerezo, 2010, pág. 65) con el fin de alcanzar un «modelo del ciudadano opendata, informado en plenitud y firmemente decidido a decidir» (Álvarez-García, Gértrudix-Barrio y Rajas-Fernández, 2014, pág. 683). Para ello, se deben crear oportunidades de aprendizaje a los estudiantes para que desarrollen destrezas a través de nuevos enfoques educativos donde la toma de decisiones empodere al estudiante. Un ejemplo de ello lo encontramos en la Universidad de Harvard, en la que los estudiantes escogen las asignaturas que conforman su programa académico y apuestan por los profesores que mejor se acomoden a sus intereses, pues estos pueden proponer nuevas materias o cambiar los contenidos cada curso académico (Torres Menárguez, 2017b; Tiching, 2014).

Evidentemente son los docentes los encargados de crear esas oportunidades de aprendizaje, pero deben estar preparados para empoderar a las nuevas generaciones con las ventajas que les aportan las TIC (UNESCO, 2008). Las simulaciones interactivas, los REAs o instrumentos de recolección y análisis de datos pueden ser los mejores recursos que permitan la asimilación de conceptos. Y es que los datos abiertos necesitan de tecnología para ser utilizados de manera eficaz, por lo que se precisa de una mayor innovación en las aulas a través de experiencias de aprendizaje que utilicen TIC (Area, Gutiérrez y Vidal, 2012). Para hacer un buen uso docente de los datos abiertos se requiere de una capacitación tecnológica de los profesores por la necesidad de conocer: (1) cómo acceder a los datos, (2) la calidad de los mismos para su explotación, y (3) cómo recuperarlos, tratarlos y analizarlos para su reutilización.

Por ello, se señala la conveniencia de incorporar en los planes de estudio de la educación universitaria una formación específica y transversal relacionada con un uso responsable y crítico de la información basada en datos abiertos de las instituciones públicas, puesto que el tratamiento y el análisis adecuado de estos datos permitiría contar con más información, lo que capacitaría para la toma de decisiones, además de fomentar una actitud crítica ante las fuentes, los datos y la información obtenida, al tiempo que implicaría el conocimiento de las administraciones públicas y la legislación relacionada con el acceso y reutilización de estos datos. 
La aportación más importante de esta investigación, a raíz de lo declarado por los docentes encuestados, es que se puede afirmar que los datos abiertos constituyen un recurso para el aprendizaje de primer orden, que permite la comprensión objetiva de la realidad y una práctica válida para el desarrollo de la innovación educativa en el contexto cambiante de nuestro tiempo. Igualmente, permiten el desarrollo de habilidades que favorecen la reflexión y la construcción del pensamiento crítico, el fomento de la autonomía para la resolución de problemas e impulsan el empoderamiento del estudiante en la toma de decisiones. Además, interesan por la fiabilidad de las fuentes de origen, la facilidad de acceso, la posibilidad de reutilización, la igualdad de oportunidades que brinda y la participación activa que fomenta. 


\section{REFERENCIAS BIBLIOGRÁFICAS}

Abero, L., Berardi, L., Capocasale, A., García Montejo, S., y Rojas Soriano, R. (2015). Investigación educativa: abriendo puertas al conocimiento. Ministerio de Educación de Perú. Recuperado de https://goo.gl/1Bfkc6

Aguilar Gavira, S., y Barroso Osuna, J. (2015). La triangulación de datos como estrategia en investigación educativa. Pixel-bit. Revista de medios y educación, 47, 73-88. http://dx.doi. org/10.12795/pixelbit.2015.i47.05

Álvarez-García, S., Gértrudix-Barrio, M., y Rajas-Fernández, M. (2014). La construcción colaborativa de bancos de datos abiertos como instrumento de empoderamiento ciudadano. Revista Latina de Comunicación Social, 69, 661-683. http://dx.doi.org/10.4185/ RLCS-2014-1029

Ananiadou, K. \& M. Claro (2009). 21st Century Skills and Competences for New Millennium Learners in OECD Countries. OECD Education Working Papers, 41, Paris, France, OECD Publishing. http://dx.doi. org/10.1787/218525261154

Area, M., Gutiérrez, A. \& Vidal, F. (2012). Alfabetización digital y competencias informacionales. Madrid: Fundación Telefónica. Recuperado de https://goo. gl/yogVWh

Area, M. y Pessoa, T. (2012). De lo sólido a lo líquido: Las nuevas alfabetizaciones ante los cambios culturales de la Web 2.0. Comunicar, 38, 13-20. https://doi. org/10.3916/C38-2012-02-01

Astigarraga, E. (2003). El método Delphi. San Sebastián: Universidad de Deusto. Recuperado de https://goo.gl/eW7UpS

Baack, S. (2015). Datafication and empowerment: How the open data move- ment re-articulates notions of democracy, participation, and journalism. Big Data \& Society, 2 (2). http://dx.doi. org/10.1177/2053951715594634

Baena Paz, G. (2014). Metodología de la investigación. México: Grupo Editorial Patria.

Bellanca, J., \& Brandt, R. (eds.) (2010). 21st Century Skills: Rethinking How Students Learn. Bloomington, IN, USA: Solution Tree.

Bisquerra, R. (2004). Metodología de la investigación educativa, vol. 1. Madrid: La Muralla, S.A

Browne, T., Holding R., Howell A., \& Rodway-Dyer, S. (2010). The challenges of OER to Academic Practice. Journal of Interactive Media in Education, (1), p.Art. 3. http://doi. org/10.5334/2010-3

Butcher, N., Kanwar, A., y UvalicTrumbic, S. (2015). Guía básica de recursos educativos abiertos (REA). París, France: UNESCO Publishing. Recuperado de https://goo.gl/wjeoYn

Cabero, J. y Llorente, M. C. (2008). La alfabetización digital de los alumnos. Competencias digitales para el siglo xxI. Revista Portuguesa de Pedagogía, 42(2), 7-28. http://doi. org/10.14195/1647-8614_42-2_1

Catalunya Dades (23 de abril de 2014). Big Data Week 2014. Recuperado de https://bit.ly/2HSRylg

Cerezo, J.M. (2010). Hacia la ciudadanía digital. Innovación para el empoderamiento ciudadano a través de las TIC. Empodera.org. Fundación Cibervoluntarios. Recuperado en: https://goo.gl/uMNfcu 
Civio (s/f). Civio. Datos que cuentan contra la opacidad. Recuperado de https://bit.ly/2FFx4tW

Chicaiza Espinosa, J. (2016). Un enfoque basado en linked data para soportar la búsqueda personalizada de recursos educativos abiertos. [Tesis doctoral]. Universidad Politécnica de Madrid. https://doi.org/10.20868/UPM. thesis.44387.

Cosculluela, A., Fornieles, A., y Turbany, J. (2009). El análisis cuantitativo de datos. Barcelona: UOC.

Daga, E., D’Aquin, M., Adamou, A., \& Brown, S. (2016). The Open University Linked Data-data. open. ac. uk. Semantic Web, 7(2), 183-191. Recuperado de https://bit.ly/2SqhgSf

Davies, T., \& Perini, F. (2016). Researching the emerging impacts of open data: revisiting the ODDC conceptual framework. The Journal of Community Informatics, 12(2). (Special issue on Open Data for Social Change and Sustainable Development), 148-178. Recuperado de https://goo.gl/w6sBTC

Datos.gob.es (23 de octubre de 2014). Decálogo del reutilizador de Datos Abiertos del Sector Público. Recuperado de https://bit.ly/2D85xy4

De Benito Crosetti, B., y Salinas Ibáñez, J. (2016). La Investigación Basada en Diseño en Tecnología Educativa. Revista Interuniversitaria de Investigación en Tecnología Educativa (RiiTE), 0, 44-59. http://dx.doi. org/10.6018/riite/2016/260631

Díez, E.J., y Millán, R.A. (2011). Open Data y RISP: generando valor social y económico. Transparencia e innovación en la eAdministración. Santiago de Compostela: Colexio Profesional de
Enxeñaría en Informática de Galicia. Recuperado de https:/goo.gl/9WxdC7

Firican, G. (8 de febrero de 2017). The 10 Vs of Big Data. Upside where Data means business. Recuperado de https:// goo.gl/XuJ9M8

Gálvez de la Cuesta, M.C. (2015) Alfabetización mediática en el ecosistema de datos abiertos: evaluación de la competencia digital de los futuros maestros de Educación Primaria. [Tesis doctoral]. Universidad Complutense de Madrid. Recuperado de http:// eprints.ucm.es/42058/1/T38621.pdf

Gertrudis-Casado, M.C., GértrudixBarrio, M., y Álvarez-García, S. (2016). Competencias informativas profesionales y datos abiertos. Retos para el empoderamiento ciudadano y el cambio social. Comunicar, 47, 39-47. http://dx.doi.org/10.3916/C472016-04

Gértrudix-Barrio, F., Gálvez de la Cuesta, M.C., Said-Hung, E., y DuránMedina, J.F. (2016). Alfabetización digital, competencias mediáticas y Open Data. RELATEC, 15(2), 113121. http://dx.doi.org/10.17398/1695288X.15.2.113

Gilmore, R.O., Adolph, K.E., Millman, D.S., \& Gordon, A. (2016). Transforming education research through open video data sharing. Advances in engineering education, 5(2). Recuperado de https://goo.gl/ rskZGG

Giráldez, A. (30 de marzo de 2017). El arte de la posibilidad: ¿Y si cambiar la escuela fuese viable? Educación 3.0. https://goo.gl/U7na9G

Guy, M. (23 de noviembre de 2014). Open (data in) education. $2^{\circ}$ International Open Data Dialog. Recuperado de https://bit.ly/2MOG4y9 
Hase, S., \& Kenyon, C. (2007) Heutagogy: A Child of Complexity Theory. Complicity: An International Journal of Complexity and Education, 4(1), 111-118. Recuperado de https:// goo.gl/eVjh3R

Hyvönen, E., Heino, E., Leskinen, P., Ikkala, E., Koho, M., Tamper, M., Tuominen, J., \& Mäkelä, E. (2016). WarSampo Data Service and Semantic Portal for Publishing Linked Open Data about the Second World War History. [paper] Recuperado de https://goo.gl/ hK6hE8

Hellberg, A.S., \& Hedström, K. (2015). The story of the sixth myth of open data and open government. Transforming Government: People, Process and Policy, 9(1), 35-51. https:// doi.org/10.1108/TG-04-2014-0013

IBM Analytics (s/f). What is Big Data? Recuperado de https://goo.gl/nxhSPC

Iglesias Fraga, A. (5 de julio de 2016). Radiografía de las TIC en las universidades españolas. TICbeat. Recuperado de https://goo.gl/xMcmgj

Insight (2011). Informe donde se analiza y compara los datos sobre la situación de las TIC en Educación en Europa. Recuperado de http://goo.gl/501D2

Klassen, T. (18 de febrero de 2013). 5 questions with David Wrate on Open Data and Higher Education. BCcampus. Recuperado de https://goo. gl/DDMzR9

Kostkova, P; Brewer, H; de Lusignan, S; Fottrell, E; Goldacre, B; Hart, G; Koczan, P; Knight, P; Marsolier, C; McKendry, RA; Ross, E; Sasse, A; Sullivan, R; Chaytor, S; Stevenson, O; Velho, R., \& Tooke, J. (2016). Who Owns the Data? Open Data for Healthcare. Front Pub- lic Health, 4(7). https://doi.org/10.3389/ fpubh.2016.00007
Manyika, J., Chui, M., Farrell, D., Van Kuiken, S., Groves, P., \& Almasi Doshi, E. (2013). Open data: Unlocking innovation and performance with liquid information. McKinsey Global Institute. Recuperado de https://goo. gl/UtT8hL

McAuley, D., Rahemtulla, H., Goulding, J., \& Souch, C. (2011). How Open Data, data literacy and Linked Data will revolutionise higher education. En: L. Coiffait (ed.) Blue Skies. New thinking about the future of higher education (pp. 8-93), London, UK: Pearson.

Mejía Giraldo, M. (2014). Open Data y Big Data, para un modelo de innovación social y sostenibilidad para Colombia. [Tesis doctoral]. Universidad Pontificia de Salamanca. Recuperado de https://goo.gl/1crGsY

Mejora tu escuela. Recuperado de https:// bit.ly/1cUPcf8

Mikroyannidis, A., Domingue, J., Maleshkova, M., Norton, B., \& Simperl, E. (2016). Teaching Linked Open Data Using Open Educational Resources. In D. Mouromtsev \& M. d'Aquin (eds). Open Data for Education, (pp. 135-152). Amsterdam, Netherlands: Springer International Publishing.

Mireles, N.P. (2012). Hacia una cultura de la apertura: concientizando y posibilitando políticas en la Educación Superior en América Latina. Revista Educación Superior y Sociedad, 16(2). Recuperado de http://goo.gl/m4z2BW

Muñoz Santamaría, L. (10 de mayo de 2016) Origen y potencia del Open Data. Recuperado de https://goo.gl/AVZQxJ

Novagob (2017). Novagob 2017 en cifras. https://goo.gl/xfws5r 
Normandeau, K. (12 de septiembre de 2013). Beyond Volume, Variety and Velocity is the Issue of Big Data Veracity. Insidebigdata.com. Recuperado de https://goo.gl/CNTiWh

Núcleo Interdisciplinario sobre Recursos Educativos Abiertos y Accesibles (22 de junio de 2016). Datos abiertos como Recursos Educativos Abiertos. Proyectar-Explorar-Narrar. Montevideo, Uruguay: Universidad de la República de Uruguay. Recuperado de https://goo.gl/zBvtky

Open Knowledge International (s/f). Open Data Handbook. Recuperado de https://bit.ly/1Nafn2S

Open Society Foundations (2007). Declaración de Ciudad del Cabo para la Educación Abierta: Abriendo la promesa de Recursos Educativos Abiertos. Recuperado de https://goo. $\mathrm{gl} / 4 \mathrm{DRZ}$

OpenKratio (2018). Recuperado de https://bit.ly/2DTQGsI

OpenCoesionSchool (2013). A Scuola di OpenCoesione. Recuperado de https:// goo.gl/nWKigm

QEdu. Use dados (s/f). Transforme a educaçao. Recuperado de https://bit. ly/1tp3Qn

IPantano, E., Priporas, C.V., \& Stylos, N. (2017). 'You will like it!' using open data to predict tourists' response to a tourist attraction. Tourism Management, 60, 430-438. https://doi. org/10.1016/j.tourman.2016.12.020

Reig, D., y Vílchez, L. F. (2013). Los jóvenes en la era de la hiperconectividad: tendencias, claves y miradas. Madrid: Fundación Telefónica y Fundación Encuentro. Recuperado de https://goo. $\mathrm{gl} / \mathrm{L} 7 \mathrm{~W} 4 \mathrm{t}$

Rijmenam, M. V. (7 de Agosto de 2013). Why The 3V's Are Not Sufficient to
Describe Big Data. Dataflop.com. Recuperado de https://goo.gl/Lkj8k5

Rivas Rebaque, B. (25 de julio de 2018). Sobre datos abiertos: definición, principios, tipos y ejemplos para educación. Ciberimaginario blog. https://ciberimaginario.es/ es/2018/07/25/sobre-datos-abiertos/

Rivas-Rebaque, B., De Cisneros de Britto, J.C. \& Gértrudix-Barrio, F. (2015). Análisis de las claves en las Políticas Educativas para el Empoderamiento Ciudadano. EDUTEC, 53. http://dx.doi. org/10.21556/edutec.2015.53.300

Rouder, J.N. (2016). The what, why, and how of born-open data. Behavior research methods, 48(3), 1062-1069

Sánchez, C.M. (2016). Stephen Heppell: «En el 2030 nos reiremos de muchas cosas de los colegios de hoy». XLSemanal. Madrid: Fundación Telefónica. Recuperado de https://goo. $\mathrm{gl} / \mathrm{mrxHcS}$

SantillanaLab (s/f). Carlos Magro nos da las claves para entender el cambio en la Educación. Recuperado de https://bit. ly/2GndaE7

Tauberer, J. (s.f). Eight principles of open government data. Recuperado de https://bit.ly/2Ao1U5Z

Tejedor, S., y Pulido, C. (2012). Retos y riesgos del uso de Internet por parte de los menores. ¿Cómo empoderarlos? Comunicar, 39, 65-72. https://doi. org/10.3916/C39-2012-02-06

Tiching (13 de marzo de 2014). Michael Jay: "Un docente debe crear deseo de aprendizaje». Tiching blog. Recuperado de http://bit.ly/Na1bdn

Torres Menárguez, A. (23 de enero de 2017a). El profesor del siglo XXI tiene que enseñar lo que no sabe. Periódico El País. Recuperado de https://goo.gl/ fHjnoy 
Torres Menárguez, A. (3 de abril de 2017b). En las aulas de Harvard ninguna pregunta se considera estúpida. Periódico El País. Recuperado de https://goo.gl/tXCZhb

Umbrich, J., Neumaier, S., \& Polleres, A. (2015). Quality assessment and evolution of open data portals. In Future Internet of Things and Cloud (FiCloud), 2015 3rd International Conference on, 404-411. Washington, USA: IEEE. https://doi.org/10.1109/ FiCloud.2015.82

UNESCO (2008). Estándares de competencias TIC para docentes. París, France: Organización de las Naciones Unidas para la Educación, la Ciencia y la Cultura. Recuperado de https://goo. gl/Sw2uQo

Weller, M., (2012). The opennesscreativity cycle in education. Journal of Interactive Media in Education (1), p.Art. 2. DOI: http://doi. org/10.5334/2012-02
Wikibooks, The Free Textbook Project from (13 de diciembre de 2014). Open Education Handbook/Open Education Data. Recuperado de https://goo.gl/ BtKpwH

Worthy, B. (2015). The impact of open data in the UK: Complex, unpredictable, and political. Public Administration, 93(3), 788-805. https:// doi.org/10.1111/padm.12166

Zapata Rojas, J. (2013). Clase invertida como metodología para mejorar las capacidades de resolver problemas en el ámbito de la formación profesional de los estudiantes de pregrado del ciclo regular en el curso de Matemáticas Básica Cero. Researchgate. Recuperado de http://goo.gl/gPcxnn

Zuiderwijk, A., \& Janssen, M. (2014). Open data policies, their implementation and impact: A framework for comparison. Government Information Quarterly, 31(1), 17-29. https://doi.org/10.1016/j. giq.2013.04.003 


\section{PERFIL ACADÉMICO Y PROFESIONAL DE LOS AUTORES}

Begoña Rivas-Rebaque. Doctora en Investigación en Humanidades, Artes y Educación por la Universidad de Castilla la Mancha. Investigadora del Grupo Ciberimaginario. Asesora Técnico Docente en el Centro de Innovación en Educación Digital: URJC online. Máster Universitario en Educación y TIC e-learning (UOC). Graduada en Magisterio en Educación Infantil y Diplomada en Magisterio de Lenguas Extranjeras por la Universidad de Castilla-La Mancha. Miembro del grupo de investigación CIBERIMAGINARIO de la URJC.

Felipe Gértrudix-Barrio. Doctor en Creatividad Aplicada por la UCM. Profesor Titular y Vicedecano en la Facultad de Educación de Toledo (UCLM). Ha sido Profesor Visitante en la Pontificia Universidad Católica de Chile, Universidad de Carazo-Managua (Nicaragua) y en UNINORTE de Barraquilla como experto TIC y educación. Miembro de los grupos de investigación SOCMEDIA de la Universidad Complutense de Madrid e IP de CIBERIMAGINARIO de la UCLM.

Julio César de Cisneros de Britto. Doctor en Ciencias Políticas por la Universidad Autónoma de Madrid. Licenciado en Sociología y Ciencias Políticas (UNED). Profesor Contratado Doctor de Sociología en la Facultad de Educación de Toledo. Especialista en Sociología de la Educación, Comunidades Virtuales y Redes Sociales. Ha sido Profesor Visitante en La Universidad Autónoma de Zacatecas (Méjico). Director de proyectos de innovación docente. Miembro del grupo de investigación CIBERIMAGINARIO de la UCLM.

Dirección de los autores: Begoña Rivas Rebaque

Centro en Innovación en Educación Digital:

URJC online

Universidad Rey Juan Carlos

C/Tulipán, s/n.

28933 Móstoles (Madrid)

E-mail: begona.rivas@urjc.es

Felipe Gértrudix Barrio

Julio César de Cisneros de Britto

Facultad de Educación de Toledo

Universidad de Castilla-La Mancha

Avda. Carlos III, s/n

45071 Toledo

E-mail: felipe.gertrudix@uclm.es

juliocesar.cisneros@uclm.es

Fecha Recepción del Artículo: 12. Febrero. 2018

Fecha Modificación del Artículo: 29. Septiembre. 2018

Fecha Aceptación del Artículo: 01. Octubre. 2018

Fecha Revisión para Publicación: 01. Febrero. 2019 
